\title{
Water harvesting from atmosphere powered by Solar cells
}

\author{
Shuai Yuan ${ }^{1, a}$, Songjing $\mathrm{Li}^{1{ }^{1, \mathrm{~b}, *}}$ and Zhiyang $\mathbf{Y u}^{1}$ \\ ${ }^{1}$ Department of Fluid Control and Automation, Harbin Institute of Technology, West Dazhi Street, Harbin, \\ China \\ a yuanshuaiHIT@outlook.com, ${ }^{\mathrm{b}}$ lisongjing@hit.edu.cn \\ *corresponding author
}

Keywords: water harvesting, solar cell, metal-organic framework

\begin{abstract}
Water is of vital importance for all species including mankind. In recent years water shortage has aroused wide concern because it is also the root of many other serious social problems, such as poverty, health problems, and national security. Water harvesting from air has long been seen as a potential solution to address this crisis. Traditional method based on air refrigeration can yield considerable water production while at the cost of tremendous electricity consumption, thus not eco-friendly. Recent studies focus on the adsorption-desorption cycle of substances with high water uptake capability, such as metal-organic frameworks (MoFs), which can exploit low grade heat resources, mainly sunlight, thus requiring no extra power input. While the working cycle of this method has been proved time consuming, typically for hours. Here a compromising low energy-consuming solution is proposed to shorten the time span of a single cycle by using ceramic heater and semiconductor chilling plate powered by solar cells. Results show that the new solution can efficiently shorten the duration of this process, thus promoting the total yield of water harvesting equipment.
\end{abstract}

\section{Introduction}

A sustainable fresh water supply is the foundation upon which all species survive. While the most majority of water on earth is in the ocean, which cannot be used directly. With the increase of population, the conflicts between human and the limited fresh water resources have been a serious endemic and global crisis. Other issues derived from water shortage, such as poverty, health problems and national security, have plagued most developing countries for a long period of time. A traditional method to solve this problem is desalination of seawater, mostly used by seashore areas. Nevertheless, this method has been proved neither cost-effective [1] nor energy-saving [2].

Another potential source of fresh water is atmosphere, even in the most drought desert [3]. The water contained in the air over land, though negligible compared to the total amount of water on earth, can be up to $3000 \mathrm{~km}^{3}$ [4]. Tremendous efforts have been made to take advantage of this untapped water resource. I. Lekouch et al. [5] investigated the potential of fog and dew as alternative sources of water, in a semi-arid area in north-west Africa. Further analysis of the collected water revealed that it could meet the criteria recommended by the World Health Organization. An intuitional method to extract water from air would be chilling humid air to dew point and collect the dewed droplets. To be sure, this method can provide significant fresh water, but requires a great deal of electricity. Meanwhile, the utilization of chlorofluorocarbons (CFCs) and hydro chlorofluorocarbons (HCFCs) as working medium in refrigeration equipment has been proved plays an inexcusable role in the depletion of ozone layer.

Alternative solutions powered by renewable energy emerge. Sunlight is available in most arid region. This idea gives rise to a variety of prototypes based on sorption cycle of water-affinity substances powered by solar energy. H.I. Abualhamayel et al. [6] proposed an experimental prototype using liquid desiccant to absorb vapor overnight and desorb vapor when heated by 
sunlight during the day. J.G. Ji et al. [7] reported a solid composite adsorbent synthesized by ultralarge pore crystalline and calcium chloride, which has an adsorption capacity as high as $1.75 \mathrm{~kg} / \mathrm{kg}$ adsorbent. You-Kyong Seo [8] investigated the water sorption properties of crystalline metalorganic frameworks (MOFs), revealing that MIL-100 and MIL-101 yield most advanced water up take capacity. More recently, Hyunho Kim [9] reported MOF-801 as adsorbent has a water uptake capability of $2.8 \mathrm{~kg} / \mathrm{kg}$ adsorbent per day at a relative humidity level as low as $20 \%$.

However, another problem lies in the aforementioned method is that a long time span (typically for hours) is required to make the adsorbent fully recovered during the desorption process. Considering most of the current methods use sunlight directly for power supply, we assumed that using solar cells as energy source to heat the adsorbent and chill the condenser while desorption would be more efficient, because the area receiving solar energy could be increased by using solar cells, thus increasing the energy input of desorption process. Though the energy efficiency could be reduced as extra converting process is introduced, this can be counterbalanced by increasing the area of solar cells.

Experiments were then conducted to verify this assumption. The adsorption-desorption system is mainly based on the prototype proposed by Hyunho Kim in preference [9]. And MOF-801 used in our experiments is synthesized by School of Chemistry and Chemical Engineering of HIT.

\section{Experiments}

\subsection{Experimental setup}

The entire system we designed is illustrated as Figure 1, followed by detailed explanations.

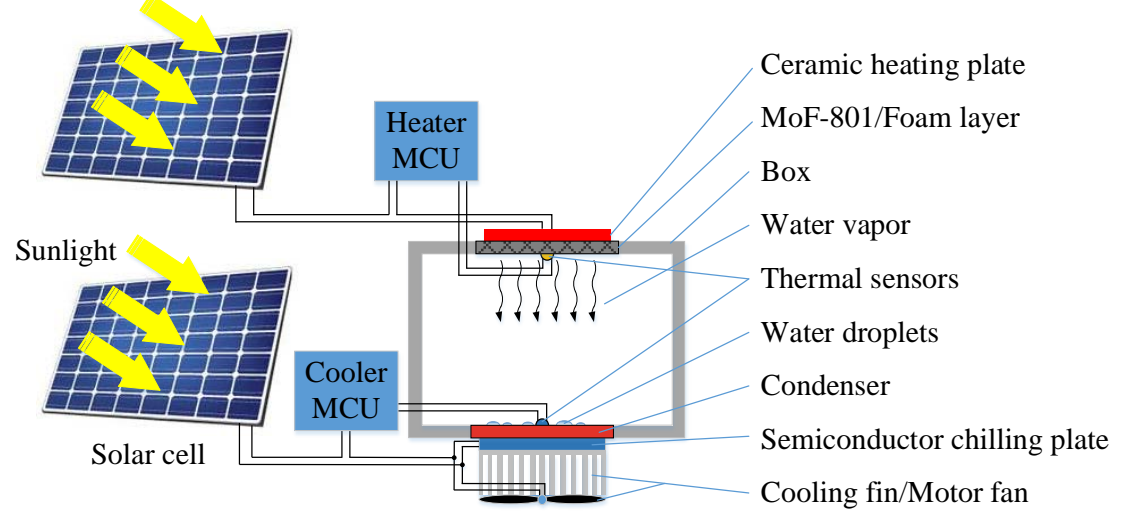

Figure 1 Illustration of the experimental system

1) Two microcontroller units (MCU) with thermal sensors (Negative temperature coefficient resistance)

Both MCUs (Heater MCU and Cooler MCU) are physically identical, with different preseted temperature thresholds of $100^{\circ} \mathrm{C}$ (normally closed) and $5^{\circ} \mathrm{C}$ (normally open), respectively. The control accuracy of both MCUs can be up to $\pm 0.1^{\circ} \mathrm{C}$. Because the main purpose of the experiment is to justify the feasibility of heater/cooler powered by solar cells, the MCUs used in this prototype are powered by DC regulated power supply $(12 \mathrm{~V})$. The integration of MCUs into the solar-cellpowered system can be easily achieved by well-designed and high efficient programming, because the power of MCUs is typically much lower than heater/cooler ( 1 W each MCU).

2) Low power consumption heater (ceramic heating plate) and cooler (semiconductor chilling plate)

Both heater and cooler are based on electrothermal effect with same rated voltage of $5 \mathrm{~V}$, and with a power of $8 \mathrm{~W}$ and 5W, respectively. This two components are shown as Figure 2. On the part of the cooler, aluminium cooling fin and a motor fan are required to ensure a good performance. Experiments carried out under room temperature condition (i.e., $20^{\circ} \mathrm{C}$, $\mathrm{RH} 65 \%$ ) demonstrate that the heater can provide a hot plane with a temperature up to $110^{\circ} \mathrm{C}$. In the experiment, the threshold of heater $\mathrm{MCU}$ is set at $100^{\circ} \mathrm{C}$, in order to prevent the MoF-801 layer from being overheated. The 
cooler can provide a $-20 \sim-10^{\circ} \mathrm{C}$ temperature difference to the object pasted to its surface. And this temperature difference depends greatly on surrounding conditions such as air temperature, wind velocity, etc. The threshold of cooler MCU is set at low temperature of $5^{\circ} \mathrm{C}$, in order to probe the potential of the cooler. Actually in the experiment the temperature of the cooler never reached $5^{\circ} \mathrm{C}$.

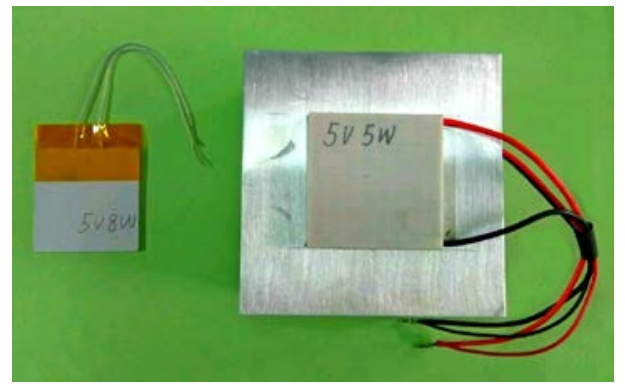

Figure 2 Heater (left) and cooler (right).

3) Solar cells

Two identical solar cells (Maximum output: 5V/10W) are deployed as energy supply of the heater and cooler, respectively. The area of each solar cell is $280 \mathrm{~mm} \times 280 \mathrm{~mm}$. A manostat and a volt ampere meter are inserted into the circuit of each cell to stabilize and indicate the output current and voltage, respectively.

4) Water sorption and condenser

A piece of aluminium foam with a porosity of $75 \%$ was cut into a layer with a dimension of $40 \mathrm{~mm} \times 40 \mathrm{~mm} \times 5 \mathrm{~mm}$. Then $2.05 \mathrm{~g}$ activated MoF-801 was infiltrated into it. The condenser is a red cupper cube with a dimension of $40 \mathrm{~mm} \times 40 \mathrm{~mm} \times 10 \mathrm{~mm}$. In order to indicate the diameter of droplets, we pasted a piece of coordinate paper to the condenser.

\subsection{Working mechanism}

There are two distinct processes during a single adsorption-desorption cycle on the part of the prototype we designed:

1) Vapour adsorption: the activated MoF-801 powder is infiltrated into an aluminium foam layer, then exposed to atmosphere overnight, during which MoF-801 becomes saturated with water vapour, because the humidity of air is remarkably higher in the evening;

2) Water desorption and condensation: during daytime, the solar cells are exposed to the sun, and the heater starts to work, releasing vapour from MoF-801 layer into the box. Meanwhile, the humidity inside the box rises, and vapour starts to condense on the condenser chilled by the cooler.

\section{Water harvesting}

All of the aforementioned components were adjusted to a normal work condition separately in laboratory and then assembled to carry out the experiment outdoor. Figure 3 shows the layout of outdoor experiment.

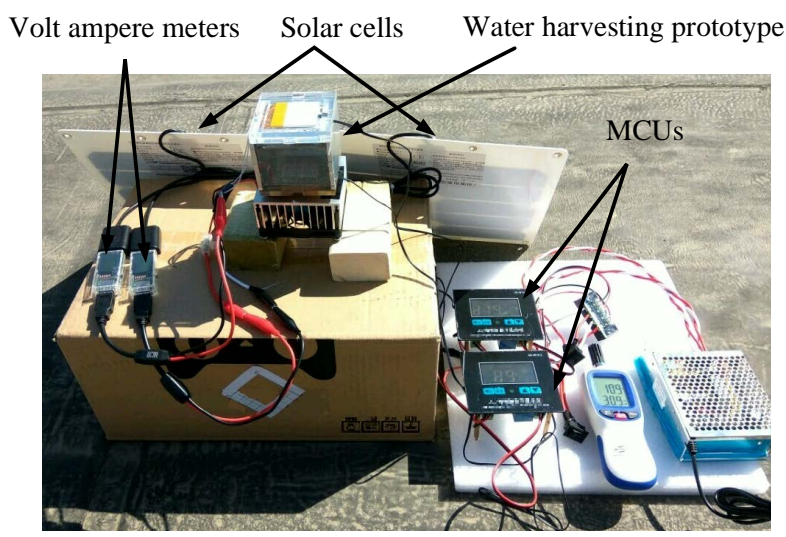

Figure 3 Layout of outdoor experiment 
The outdoor experiment was conducted on Sep.16, starting with exposing the MoF-801/foam layer in atmosphere overnight, the weight of which was measured once per hour. The weighing process was terminated at 11:00, because the foam layer was moved into the box at that time, which made it difficult to weigh the layer. Figure 4 shows the variation of the mass of MoF-801 over time.

Then during the day time, from 11:00 16:00, the solar cells were exposed perpendicular to sunlight to generate electricity to power the heater and cooler.

The atmospheric conditions, including air temperature, air humidity and dew point during the entire experiment process, are indicated in Figure 5. The voltage, current and the power of each solar cell are shown in Figure 6.

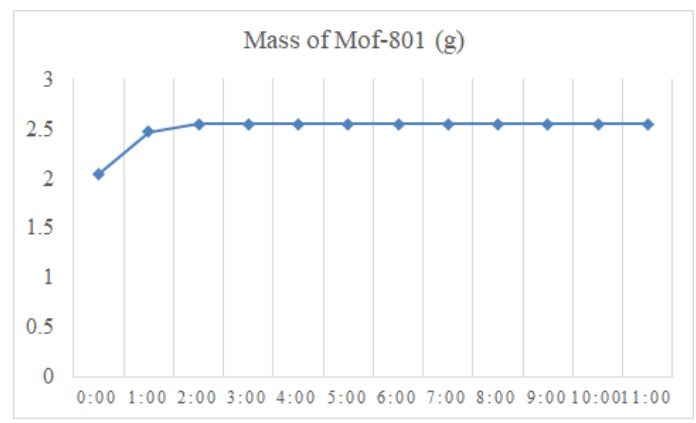

Figure 4 Variation of the mass of MoF-801 during the experiment (the mass of foam layer is deducted).

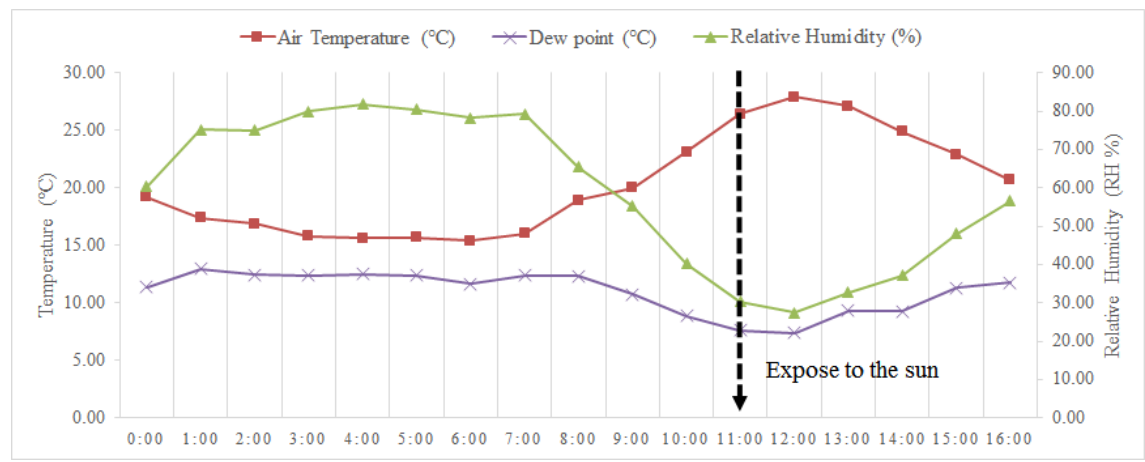

Figure 5 Temperature and dew point during the experiment. Vertical dash line indicates the time when solar cells exposed to the sun.
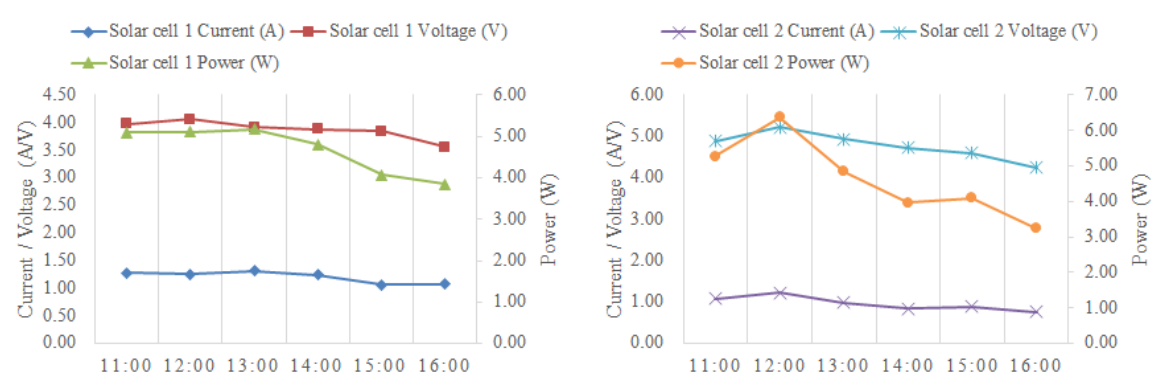

Figure 6 Current, voltage and power of solar cell 1 (heater) and solar cell 2 (cooler).

\section{System performance}

\subsection{Water harvesting performance}

From the water uptake curve of MoF-801/foam layer shown in Figure 4, we confirmed the water harvesting capability of MoF-801 during a single adsorption-desorption cycle $(0.24 \mathrm{~kg} / \mathrm{kg}$ MoF-801), which in accordance well with reference [9]. The sorbent was saturated within 2 hours, because of the high humidity ( RH 80\%).

Furthermore, after 15min exposed to the sun (i.e., 11:15), tiny water droplets could be observed ubiquitously on the surface of condenser, indicating the water condensation process began. After 
about 45min (i.e., 11:45), the distribution of droplets became stable, shown as Figure 7. In order to make it easier to estimate the mass of water on the surface of condenser, we use the following simplifications:

I All the droplets have an average diameter;

II The droplets cover the surface densely;

III A droplet is regarded as part of a sphere.

Based on the coordinate paper, we can get the approximate average diameter, about $D=0.66 \mathrm{~mm}$. The contact angle between water and the surface of coordinate paper (BOPP membrane) is $\theta=105^{\circ}$. The volume of a single droplet is:

$$
V=\pi H^{2}\left(\mathrm{R}-\frac{H}{3}\right)=\frac{\pi}{3}\left(\frac{D}{2}+\mathrm{h}\right)^{2}(D-h)
$$

Where $\mathrm{h}$ is the height of the centre of the sphere:

$$
\mathrm{h}=\frac{D}{2} \sin \left(\theta-\frac{\pi}{2}\right)
$$

According to the size of the surface of the condenser, the total quantity of droplets is:

$$
\mathrm{Q}=\left(\frac{a}{D}\right)^{2}
$$

Where $a$ is the length of the condenser.

Then we can get the mass of water, $m=\rho V Q=0.381 \mathrm{~g}$, i.e., approximately $0.381 \mathrm{~g}$ water condensed on the condenser.
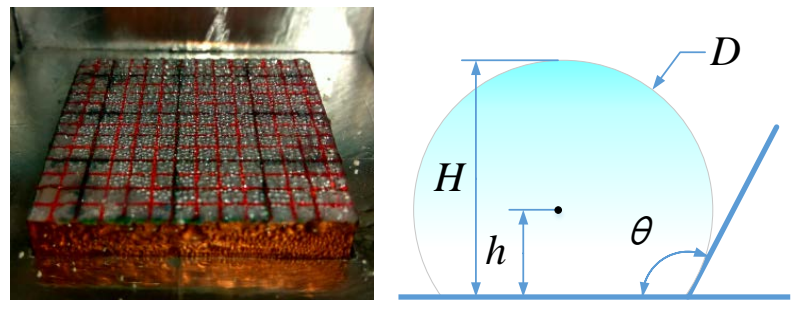

Figure 7 The droplets on the condenser at 11:45 (left); an illustration of a single droplet (right).

We noticed that some droplets condensed on the upper inner surface of the box, because the humidity is higher in the upper stratum inside the box, thus the dew point lower.

\subsection{Performance of heater, cooler and solar cells}

The heater can accelerate desorption process remarkably because the temperature of MoF-801 layer cannot rise to $100{ }^{\circ} \mathrm{C}$ if heated by sunlight directly under current air conditions. It should be noted that the temperature of MoF-801 layer during desorption process is not the higher the better. Our preliminary simulation indicates that a high temperature of MoF-801 layer could hinder the condensation process, because the isothermal curves near condenser would become thicker, thus increasing the heat dissipation requirement of condenser.

The cooler can help maintain the condenser at low temperature (near dew point), which can promote the condensation process. During the outdoor experiment, the temperature of cooler and condenser varied largely depending on the surrounding conditions, but roughly fluctuated near the dew point of surrounding atmosphere. Figure 8 illustrates heater/cooler temperature and dew point after the cells exposed to the sun.

From the power date shown in Figure 6, we notice that the solar cells didn't provide full power output, perhaps partly because of the cloudy weather. The outdoor experiment ended at about 16:00 since the voltage of solar cells declined dramatically. Nevertheless, the power output of both cells can match to the power requirement of heater and cooler. A higher power cooler could constantly chill the condenser to a temperature below dew point, but requires larger solar cells, thus increasing 
the total volume and cost of the device. In order to design an ideal cooler, power consumption, solar energy potential, energy conversion efficiency and the total cost should be taken into consideration. Our further investigation would focus on an optimization of this solar cell powered heating/chilling system.

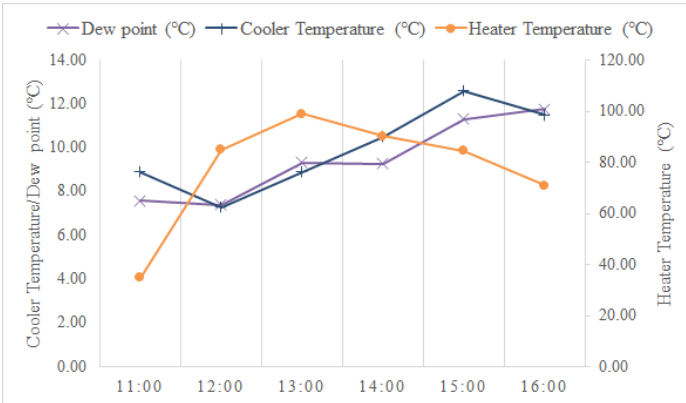

Figure 8 Detailed illustration of heater/cooler temperature and dew point.

\section{Conclusion}

Our experimental system using solar cells as energy supply to power a water harvesting prototype has shown that solar energy can be harnessed more controllable by using solar cells, and make water harvesting equipment less vulnerable to fluctuation of surrounding conditions. Meanwhile, heater and cooler can be used to accelerate the desorption process, which can help shorten the desorption process, thus promoting the total yield of equipment. Further, a higher temperature difference between the MoF-801 layer and the condenser make it possible to harvest water from drier air, thus extending the application of the device.

Limited by the vast spatial and temporal differences of vapour distribution, and the scarcity of water content in the atmosphere, water harvesting from air powered by sunlight can hardly meet the requirement of domestic or industrial usage, at least for now. While an optimistic design of our prototype can be used at other scenarios with smaller water demand per unit time, such as trickle irrigation in afforestation and agriculture.

\section{References}

[1] Ziolkowska J R. Is Desalination Affordable?-Regional Cost and Price Analysis[J]. Water Resources Management, 2015, 29(5):1385-1397.

[2] Elimelech M, Phillip W A. The future of seawater desalination: energy, technology, and the environment.[J]. Science, 2011, 333(6043):712.

[3] Cereceda P, Larrain H, Osses P, et al. The spatial and temporal variability of fog and its relation to fog oases in the Atacama Desert, Chile[J]. Atmospheric Research, 2008, 87(3):312-323.

[4] Oki T, Kanae S. Global hydrological cycles and world water resources[J]. Science, 2006, 313(5790):1068-72.

[5] Lekouch I, Muselli M, Kabbachi B, et al. Dew, fog, and rain as supplementary sources of water in south-western Morocco[J]. Energy, 2011, 36(4):2257-2265.

[6] Abualhamayel H I, Gandhidasan P. A method of obtaining fresh water from the humid atmosphere[J]. Desalination, 1997, 113(1):51-63.

[7] Ji J G, Wang R Z, Li L X. New composite adsorbent for solar-driven fresh water production from the atmosphere[J]. Desalination, 2007, 212(1-3):176-182.

[8] Seo Y K, Yoon J W, Lee J S, et al. Energy-efficient dehumidification over hierachically porous metal-organic frameworks as advanced water adsorbents[J]. Advanced Materials, 2012, 24(6):806.

[9] Kim H, Yang S, Rao S R, et al. Water harvesting from air with metal-organic frameworks powered by natural sunlight.[J]. Science, 2017, 356(6336):430. 